\title{
Estimativa das necessidades energéticas em pacientes com doença renal crônica
}

\author{
Estimating the energy requirement of \\ chronic kidney disease patients
}

Juliana Cordeiro Dias RODRIGUES'

Fernando LAMARCA ${ }^{1}$

Cecilia Lacroix OLIVEIRA2

Carla Maria AVESANI²

RE S U M O

Estabelecer as necessidades energéticas de pacientes com doença renal crônica é importante para que se possam tratar os distúrbios nutricionais encontrados nessa população. Segundo os guias de condutas voltados ao cuidado nutricional de pacientes com essa doença, a recomendação energética pode variar entre 30 e $40 \mathrm{kcal} / \mathrm{kg} / \mathrm{dia}$. Contudo, trabalhos que avaliaram os componentes do gasto energético nos pacientes com doença renal crônica sugerem que as necessidades energéticas dessa população podem diferir do valor recomendado acima, a depender da condição clínica (presença de comorbidades), da modalidade de tratamento empregado e do nível de atividade física. Dessa forma, o presente trabalho tem como objetivo fazer uma revisão dos estudos sobre o gasto energético de pacientes com doença renal crônica, com o intuito de abordar as seguintes questões: (1) as atuais recomendações de energia para pacientes com doença renal crônica estão adequadas? (2) qual equação de predição poderia ser empregada para estimar as necessidades energéticas desse grupo de pacientes? Assim, esta revisão busca auxiliar o nutricionista ao estimar as necessidades energéticas de pacientes com doença renal crônica.

Palavras-chave: Doença renal crônica. Metabolismo energético. Necessidade energética.

A B S T R A C T

Estimating the energy requirement of patients with chronic kidney disease is highly important for treating the nutritional disorders often seen in this population. According to the specific guidelines for patients with chronic kidney disease, the currently recommended daily energy intake varies from 30 to $40 \mathrm{kcal} / \mathrm{kg} / \mathrm{day}$. However,

\footnotetext{
${ }^{1}$ Nutricionistas, Universidade do Estado do Rio de Janeiro, Curso de Pós-Graduação em Alimentação, Nutrição e Saúde. Rio de Janeiro, RJ, Brasil.

2 Universidade do Estado do Rio de Janeiro, Instituto de Nutrição, Departamento de Nutrição Aplicada. R. São Francisco Xavier, 524, $12^{\circ}$ andar, sala 12025 D, Maracanã, 20559-900. Rio de Janeiro, RJ, Brasil. Correspondência para/Correspondence to: CM AVESANI.E-mails:<carla.avesani@carrenho.com.br>; <carla@carrenho.com.br>; <jubsjubs2004@yahoo.com.br>.
} 
98 | JCD RODRIGUES et al.

studies that investigated energy expenditure components of patients with chronic kidney disease suggested that the energy requirement may differ from the one proposed earlier, depending on overall clinical condition (i.e. presence of comorbidities), treatment modality and level of physical activity. With this perspective in mind, the present study aims to review the studies assessing energy expenditure focusing on two themes: (1) is the current energy recommendation for patients with chronic kidney disease adequate? (2) which predictive equation can be used when estimating the energy requirements of this population? Therefore, this review aims to help dietitians to estimate the energy needs of chronic kidney disease patients.

Indexing terms: Chronic kidney disease. Energy metabolism. Energy requirement.

\section{N T R O D U Ç Ã O}

Pacientes com Doença Renal Crônica (DRC) frequentemente enfrentam alterações do estado nutricional, dentre as quais se destacam a desnutrição e o sobrepeso/obesidade. A prevalência da primeira é variável e pode estar presente entre $18 \%$ e $70 \%$ dos pacientes com DRC, a depender da população estudada (exemplo: estágio da doença, terapia dialítica empregada no tratamento, etnia, dentre outros fatores) e do critério adotado para o diagnóstico nutricional ${ }^{1-4}$.

Embora pareça contraditório, a prevalência de sobrepeso/obesidade também é elevada, estando presente entre $20 \%$ e $60 \%$ dos pacientes com DRC ${ }^{5,6}$. Um fato que difere as duas condições é que a desnutrição pode se desenvolver ao longo da doença em razão das diversas alterações metabólicas inerentes à DRC e ao tratamento dialítico ${ }^{4}$, enquanto o sobrepeso e a obesidade podem estar presentes no início da terapia de substituição renal e não como consequência da mesma. Tal achado vem chamando atenção desde a publicação de Kramer et al. ${ }^{6}$, que demonstraram aumento na prevalência de sobrepeso e de obesidade em pacientes iniciando diálise entre 1995 e 2002, em razão da epidemia mundial de obesidade. Independentemente da causa desses distúrbios, ambos merecem tratamento adequado, com o intuito de manter e ou recuperar o estado nutricional. Para tanto, é importante estimar adequadamente as necessidades energéticas desses pacientes.

No ano de 2000, o guia norte-americano de condutas para pacientes com DRC (National Kidney Foundation - Kidney Disease Outcome
Quality Initiative - NKF/KDOQI) publicou um documento referente aos cuidados nutricionais voltados a esse grupo de pacientes ${ }^{2}$. Baseando-se nos estudos que investigaram o gasto energético dessa população, o NKF/KDOQI recomendou $35 \mathrm{kcal} / \mathrm{kg} /$ dia para pacientes com menos de 60 anos, e de 30 a $35 \mathrm{kcal} / \mathrm{kg} /$ dia para aqueles com idade $\geq 60$ anos $^{2}$. Posteriormente, foram publicados outros guias também voltados ao cuidado nutricional de pacientes com DRC ${ }^{7-9}$, sendo que a recomendação de energia se assemelhou à do NKF/KDQI, variando entre 30 e 40kcal/kg/dia 2,7-9 (Tabela 1). Dentre esses guias, o NKF/KDOQI se mantém o mais empregado por nutricionistas norte-americanos ${ }^{10}$.

Esta revisão tem como objetivo reunir estudos que tenham investigado o gasto energético de pacientes com DRC nos estágios 2 a 5 da doença (fase não dialítica e dialítica da DRC), com o intuito de abordar duas questões relevantes no tratamento clínico de pacientes com DRC: (1) as atuais recomendações de energia para pacientes com DRC estão adequadas? e (2) na prática clínica, qual equação de predição deve ser empregada para estimar as necessidades energéticas desse grupo de pacientes?

\section{Definição de necessidade e recomendação de energia}

Necessidades e recomendações de energia são termos que, embora importantes, são por vezes empregados de maneira incorreta. A necessidade nutricional pode ser definida como a quantidade de energia e de nutrientes disponíveis nos alimentos que indivíduos sadios devem ingerir 
Tabela 1. Recomendação de energia preconizada por guias de condutas específicos para pacientes com doença renal crônica.

\begin{tabular}{|c|c|c|c|c|}
\hline & \multicolumn{4}{|c|}{ Recomendação de energia } \\
\hline & NKF/KDOQI & CARI & EBPG & ESPEN \\
\hline \multicolumn{5}{|l|}{ Pré-Diálise } \\
\hline & $<60$ anos & $35 \mathrm{kcal} / \mathrm{kg}^{\mathrm{b}} / \mathrm{dia}$ & - & - \\
\hline & $35 \mathrm{kcal} / \mathrm{kg}^{\mathrm{a}} / \mathrm{dia}$ & & & \\
\hline & $\geq 60$ anos & $30-35 \mathrm{kcal} / \mathrm{kg}^{\mathrm{b}} / \mathrm{dia}^{\mathrm{c}}$ & - & - \\
\hline & $30-35 \mathrm{kcal} / \mathrm{kg}^{\mathrm{a}} / \mathrm{dia}$ & & & \\
\hline \multicolumn{5}{|l|}{ Hemodiálise } \\
\hline & $<60$ anos & - & $30-40 \mathrm{kcal} / \mathrm{kg}^{\mathrm{b}} / \mathrm{dia}^{\mathrm{e}}$ & $35 \mathrm{kcal} / \mathrm{kg} / \mathrm{dia}^{\mathrm{e}}$ \\
\hline & $35 \mathrm{kcal} / \mathrm{kg} / \mathrm{dia}^{\mathrm{a}}$ & & & \\
\hline & $\geq 60$ anos & - & - & - \\
\hline & $30-35 \mathrm{kcal} / \mathrm{kg}^{\mathrm{a}} / \mathrm{dia}$ & & & \\
\hline \multicolumn{5}{|l|}{ Diálise Peritoneal } \\
\hline & $<60$ anos & - & - & $35 \mathrm{kcal} / \mathrm{kg} / \mathrm{dia}^{\mathrm{d}, \mathrm{e}}$ \\
\hline & $35 \mathrm{kcal} / \mathrm{kg}^{\mathrm{a}} / \mathrm{dia}^{\mathrm{d}}$ & & & \\
\hline & $\geq 60$ anos & - & - & - \\
\hline & $30-35 \mathrm{kcal} / \mathrm{kg}^{\mathrm{a}} / \mathrm{dia}^{\mathrm{d}}$ & & & \\
\hline
\end{tabular}

aPeso ajustado quando a adequação do peso atual/ideal é <95\% ou >115\%; bPeso ideal; ' Indivíduos sedentários ou idosos; ${ }^{\mathbf{d}} \mathrm{A}$ energia proveniente da absorção de glicose do dialisato deve estar incluída nesse cálculo; 'Para indivíduos adultos e idosos.

NKF/KDOQI: National Kidney Foundation/Kidney Disease Outcomes Quality Initiative; CARI: Caring for Australasians with Renal Impairment; EBPG: European Best Practice Guideline; ESPEN: European Society For Clinical Nutrition And Metabolism.

para satisfazer todas as suas necessidades fisiológicas ${ }^{11}$. Já a recomendação nutricional compreende a quantidade de energia e de nutrientes que a alimentação usual deve conter para satisfazer as necessidades de quase todos os indivíduos de uma população sadia"1.

Contudo, deve-se distinguir a recomendação de energia da recomendação de nutrientes. A recomendação de energia visa satisfazer a necessidade da média da população - e não da maioria dos indivíduos -, estimada pela média ingerida, acrescida de 2 desvios-padrão, como no caso da recomendação de nutrientes ${ }^{11}$. A recomendação de energia visa, portanto, atender à necessidade individual, de forma a evitar o desenvolvimento de sobrepeso/obesidade. Nesse sentido, vale descrever a definição de necessidade esti-mada de energia, proposta pela Ingestão Dietética de Referência (Dietary Reference Intake - DRI): "ingestão média de energia suficiente para manter balanço energético de um indivíduo saudável com determinada idade, sexo, peso, estatura, atividade física e com bom estado de saúde"12. Logo, é importante conhecer sobre o balanço energético de determinada população para que as necessidades energéticas sejam estabelecidas.

No que diz respeito a pacientes com DRC, embora poucos estudos tenham avaliado o balanço energético, há uma gama de trabalhos que investigaram o Gasto Energético de Repouso (GER) e a ingestão energética dessa população.

\section{Gasto energético: componentes e métodos de aferição/predição}

O Gasto Energético Total (GET) pode ser compreendido pelo somatório da Taxa Metabólica Basal (TMB), do efeito térmico dos alimentos e do efeito térmico da atividade física. Dentre esses componentes, a TMB destaca-se por contribuir com $60 \%$ a $75 \%$ do GET. A contribuição dos demais componentes para o GET é de $10 \%$ para o efeito térmico dos alimentos, e de 15\% a 30\% para o gasto energético para atividade física ${ }^{11,12}$.

A taxa metabólica basal pode ser definida como a energia mínima despendida para manter 
as atividades celulares, teciduais, circulatórias, respiratórias, gastrointestinais e renais. Contudo, a TMB não deve ser confundida com a taxa de metabolismo de repouso, pois, apesar de ambas serem aferidas em condições semelhantes, a estimativa desta última permite que o indivíduo se desloque até o local do exame e, dessa forma, encontra-se $10 \%$ a $20 \%$ maior em relação à $\mathrm{TMB}^{11,12}$. Como as condições de medida da taxa de metabolismo de repouso são menos restritas, ela é aferida pela maioria dos estudos. Ao se extrapolar a taxa de metabolismo de repouso para 24 horas, obtém-se o gasto energético de repouso ${ }^{11,12}$.

O efeito térmico do alimento é definido pelo aumento do gasto energético decorrente dos processos de ingestão, digestão, absorção, transporte, metabolização e armazenamento dos nutrientes. Sabe-se que o efeito térmico do alimento sofre influência da quantidade e da qualidade da composição do produto consumido. Já o efeito térmico da atividade física é compreendido pelo consumo de oxigênio após o exercício, o qual varia de acordo com a intensidade e duração do exercício. Dentre os componentes do GET, o efeito térmico da atividade física é aquele que pode apresentar maior variação, tanto intraindividual quanto de um indivíduo para outro ${ }^{11,12}$.

O gasto energético de repouso pode ser aferido por métodos mais precisos (como a calorimetria direta e indireta), pela água duplamente marcada, ou ainda por métodos indiretos, como por meio de sensores de movimento, medida da frequência cardíaca e avaliação da ingestão energética. Equações de predição também são empregadas para esse fim, principalmente no contexto ambulatorial $^{12}$.

São poucos os trabalhos que analisaram o GET de pacientes com DCR por métodos indiretos, como os sensores de movimento. Já o GER foi investigado pela calorimetria indireta em vários trabalhos, incluindo pacientes na fase não dialítica e dialítica, além de estudos que também avaliaram o desempenho das equações de predição para estimar o GER dessa população (Quadro 1).

\section{Gasto energético de repouso na doença renal crônica}

O gastro energético total avaliado por calorimetria indireta vem sendo estudado há mais de duas décadas na população de pacientes com DRC (Quadro 1).

Os primeiros estudos que compararam o GER de pacientes com DRC na fase não dialítica e dialítica com o de indivíduos saudáveis não mostraram diferenças entre ambos os grupos ${ }^{13,14}$.

Posteriormente, trabalhos com objetivo semelhante, mas que empregaram metodologia mais adequada (grupos pareados por sexo e idade, e ajuste do GER pela massa corporal magra) mostraram resultados diferentes ${ }^{15-17}$. Na fase não dialítica da DRC, um estudo que avaliou o GER de indivíduos idosos ( $n=15$, idade $\geq 60$ anos) mostrou que ele se encontrava reduzido quando comparado ao de idosos saudáveis ${ }^{17}$. Corroborando esse achado, Avesani et al. ${ }^{18}$, ao analisarem o GER de indivíduos com DRC em tratamento conservador em uma amostra maior $(n=45)$ e com idade mais abrangente (33 a 56 anos), também encontraram o mesmo resultado. Trabalhos incluindo pacientes em diálise mostram resultados controversos. Ikizler et al. ${ }^{15}$, ao avaliarem o GER de pacientes em Hemodiálise (HD), encontraram que esse índice era significantemente maior nesse grupo do que em controles saudáveis, enquanto outros dois trabalhos não mostraram diferenças entre os grupos ${ }^{19,20}$. Estes últimos achados, divergentes, podem ser explicados pela característica da amostra, composta por indivíduos de faixa etária inferior (de 28 a 54 anos e de 31 a 57 anos, respectivamente) e em condições clínicas estáveis $^{19,20}$.

Ao compilar trabalhos que avaliaram o papel de comorbidades (diabetes Mellitus, hiperparatireoidismo secundário e condição inflamatória crônica) sobre o GER, observou-se que ele, nessas condições, encontrava-se aumentado em cerca de $12 \%$ a $23 \% \%^{5,16,21-23}$. Vale mencionar ainda que o próprio procedimento de HD também é capaz de aumentar o gasto energético. Ikizler et al. ${ }^{24}$, ao avaliarem o gasto energético antes, du- 
Quadro 1. Principais trabalhos que avaliaram o gasto energético de repouso pela calorimetria indireta em pacientes com doença renal crônica.

\begin{tabular}{|c|c|c|c|c|}
\hline Autores & População (n) & Pareamento & Principal objetivo & Principal achado \\
\hline Monteon et al. ${ }^{13}$ & $\begin{array}{l}12 \text { saudáveis } \\
10 \text { Cons } \\
16 \mathrm{HD}\end{array}$ & Não & $\begin{array}{l}\text { Comparar o GER, ETA e GEAF } \\
\text { de pacientes com DRC com o } \\
\text { de indivíduos saudáveis. }\end{array}$ & $\begin{array}{l}\text { O GER, ETA e GEAF de pacientes } \\
\text { com DRC (Cons e HD) são iguais aos } \\
\text { de indivíduos saudáveis. }\end{array}$ \\
\hline Schneeweiss et al. ${ }^{14}$ & $\begin{array}{l}18 \text { IRA comsepticemia } \\
11 \text { IRA sem septicemia } \\
17 \text { Cons } \\
15 \text { Azotemia severa } \\
25 \text { HD } \\
24 \text { saudáveis }\end{array}$ & $\operatorname{sim}$ & $\begin{array}{l}\text { Mensurar o GER e a oxidação } \\
\text { de substratos em diversas fa- } \\
\text { ses da doença renal. }\end{array}$ & $\begin{array}{l}\text { Pacientes com IRA e septicemia } \\
\text { apresentaram GER maior do que de } \\
\text { indivíduos saudáveis. Os demais } \\
\text { grupos apresentaram GER similar ao } \\
\text { de indivíduos saudáveis. }\end{array}$ \\
\hline Ikizler et al..$^{15}$ & $\begin{array}{l}10 \mathrm{HD} \\
10 \text { saudáveis }\end{array}$ & Sim & $\begin{array}{l}\text { Avaliar se o procedimento } \\
\text { dialítico aumenta o GE. Com- } \\
\text { parar o GER de pacientes em } \\
\text { HD com o de indivíduos sau- } \\
\text { dáveis. }\end{array}$ & $\begin{array}{l}\text { O procedimento dialítico aumenta } \\
\text { o GE, principalmente nas } 2 \text { primei- } \\
\text { ras horas de diálise. O GER foi } \\
\text { significantemente maior no grupo } \\
\text { HD. }\end{array}$ \\
\hline Avesani et al. ${ }^{16}$ & $\begin{array}{l}24 \text { DRC com DM } \\
24 \text { DRC sem DM }\end{array}$ & Sim & $\begin{array}{l}\text { Comparar o GER de pacientes } \\
\text { com DRC em Cons com e sem } \\
\text { DM. }\end{array}$ & $\begin{array}{l}\text { O GER foi maior nos pacientes com } \\
\text { DRC e DM. }\end{array}$ \\
\hline O'Sullivan et al. ${ }^{17}$ & $\begin{array}{l}15 \text { Cons } \\
15 \text { saudável }\end{array}$ & Sim & $\begin{array}{l}\text { Comparar o GER de pacientes } \\
\text { idosos em Cons com o de ido- } \\
\text { sos saudáveis. }\end{array}$ & $\begin{array}{l}\text { O GER foi menor nos pacientes em } \\
\text { Cons. }\end{array}$ \\
\hline Ikizler et al..$^{24}$ & $11 \mathrm{HD}$ & - & $\begin{array}{l}\text { Examinar o efeito da HD so- } \\
\text { bre o metabolismo de CHO, } \\
\text { PTN e LIP. }\end{array}$ & $\begin{array}{l}\text { O procedimento de HD aumentou } \\
\text { o GE, e o catabolismo proteico du- } \\
\text { rante e até } 2 \mathrm{~h} \text { após o seu término. }\end{array}$ \\
\hline Cuppari et al. ${ }^{21}$ & $\begin{array}{l}15 \text { HD HPT severo } \\
15 \text { HD HPT leve a moderado } \\
15 \text { saudáveis }\end{array}$ & $\operatorname{sim}$ & $\begin{array}{l}\text { Testar a hipótese de que HPT } \\
\text { severo pode aumentar o GER } \\
\text { de pacientes em HD e de que } \\
\text { a PTX pode reverter essa con- } \\
\text { dição. }\end{array}$ & $\begin{array}{l}\text { O GER foi maior nos indivíduos com } \\
\text { HPT severo, e a PTX reduziu o GER. }\end{array}$ \\
\hline Wang et al..23 & 251 CAPD & Não & $\begin{array}{l}\text { Determinar se o maior GER se } \\
\text { associa com pior desfecho clí- } \\
\text { nico. }\end{array}$ & $\begin{array}{l}\text { O aumento do GER se associou com } \\
\text { inflamação, função renal residual, } \\
\text { DCV, desnutrição e com maior taxa } \\
\text { de mortalidade. }\end{array}$ \\
\hline Avesani et al. ${ }^{18}$ & 91 Cons & Não & $\begin{array}{l}\text { Avaliar se o nível de função } \\
\text { renal e a presença de inflama- } \\
\text { ção crônica subclínica se asso- } \\
\text { ciam com o GER. }\end{array}$ & $\begin{array}{l}\text { O GER não se associou com o nível } \\
\text { de função renal. Pacientes com in- } \\
\text { flamação subclínica apresentaram } \\
\text { GER maior do que daqueles não in- } \\
\text { flamados. }\end{array}$ \\
\hline Utaka et al..22 & $\begin{array}{l}132 \text { Cons } \\
10 \text { com inflamação }\end{array}$ & Não & $\begin{array}{l}\text { Investigar a relação entre in- } \\
\text { flamação e o GER de pacien- } \\
\text { tes em Cons. }\end{array}$ & $\begin{array}{l}\text { O GER foi maior nos pacientes com } \\
\text { inflamação. } \\
\text { O tratamento da condição inflama- } \\
\text { tória reduziu o GER. }\end{array}$ \\
\hline Kamimura et al. ${ }^{20}$ & $\begin{array}{l}55 \text { HD } \\
55 \text { saudáveis }\end{array}$ & Sim & $\begin{array}{l}\text { Avaliar o GER e seus deter- } \\
\text { minantes nos pacientes em HD. }\end{array}$ & $\begin{array}{l}\text { O GER de pacientes em HD foi igual } \\
\text { ao dos indivíduos saudáveis. }\end{array}$ \\
\hline Kamimura et al. ${ }^{5}$ & $\begin{array}{l}80 \mathrm{HD} \\
30 \mathrm{HD} \text { citocinas } \\
11 \text { saudáveis }\end{array}$ & & $\begin{array}{l}\text { Investigar a relação entre a } \\
\text { citocina pro-inflamatória (IL-6) } \\
\text { e o GER de pacientes em HD. }\end{array}$ & $\begin{array}{l}\text { IL-6 sérica encontrou-se associada } \\
\text { com aumento do GER em pacien- } \\
\text { tes em HD. }\end{array}$ \\
\hline
\end{tabular}

ETA: Efeito Térmico do Alimento; GE: Gasto Energético; GEAF: Gasto Energético para Atividade Física; GER: Gasto Energético de Repouso; Cons: Tratamento Conservador; HD: Hemodiálise; IRA: Insuficiência Renal Aguda; CAPD: Diálise Peritoneal Ambulatorial Contínua; DP: Diálise Peritoneal; DM: Diabetes Mellitus; DCV: Doença Cardiovascular; CHO: Carboidrato; PTN: Proteína; LIP: Lipídeo; HPT: Hiperparatireoidismo; PTX: Paradectomia; IL-6: interleucina 6. 
102 JCD RODRIGUES et al.

rante e após a sessão de diálise, observaram aumento de $7 \%$ no gasto energético durante o procedimento dialítico, e de $12 \%$ após 2 horas de seu término. Em conjunto, esses trabalhos mostram que, dependendo do estágio da DRC (não dialítico ou dialítico) e da condição clínica do paciente (presença ou não de comorbidades), o GER pode se encontrar reduzido, igual ou superior ao de indivíduos saudáveis.

\section{Efeito térmico dos alimentos e gasto energético para atividade física na DRC}

Apesar de o GER ser o principal contribuinte do GET, o gasto energético proveniente do efeito térmico do alimento e da atividade física é um componente que também deve ser estudado nos pacientes com DRC, uma vez que juntos perfazem de $25 \%$ a $40 \%$ do $\mathrm{GET}^{12}$.

Com relação ao efeito térmico dos alimentos, somente um estudo avaliou esse tópico em pacientes com DRC. Monteon et al. ${ }^{13}$ demonstraram por meio de calorimetria indireta, que a curva de variação do gasto energético após ingestão de uma refeição padrão não foi diferente entre pacientes renais crônicos e indivíduos saudáveis, assim como também não diferiu entre pacientes na fase não dialítica da DRC e na fase dialítica. Após a refeição, observou-se primeiramente (após 15 minutos) um aumento no gasto energético, seguido por um plateau (após 15 a 30 minutos) e posterior redução do gasto energético (após 30 a 45 minutos), sendo que este não retornou ao valor basal após 4 horas da refeição ${ }^{13}$. Embora apenas um trabalho tenha feito essa avaliação, esse resultado sugere que o efeito térmico dos alimentos não se encontra alterado na DRC.

A avaliação do gasto energético para atividade física na DRC também é restrita a um número pequeno de estudos ${ }^{13,25-28}$, dos quais apenas um empregou a calorimetria indireta ${ }^{13}$. Neste estudo, demonstrou-se um aumento progressivo do gasto energético conforme se aumentava a carga de resistência imposta durante o exercício (bicicleta ergométrica), sendo este incremento se- melhante entre os grupos estudados: pacientes na fase não dialítica, em HD e indivíduos saudáveis.

Os demais trabalhos que avaliaram o gasto energético para atividade física na DRC o fizeram por meio de sensores de movimento em pacientes em HD ${ }^{25-28}$. Observou-se que esse componente apresentou grande variação tanto intra quanto inter-individual ${ }^{25-27}$. Ademais, ao comparar o gasto energético para atividade física entre o dia com e sem tratamento dialítico, notou-se que ele foi significantemente menor no dia com diálise ${ }^{25-27}$. A menor atividade física observada no dia de diálise possivelmente resulta do tempo que os pacientes passam sentados para o procedimento dialítico (cerca de 4 horas/sessão de diálise).

Outro achado comum nos estudos é o reduzido gasto energético para atividade física. Baria et al..$^{25}$ demonstraram que o gasto energético para atividade física nos pacientes em HD era significantemente menor que o de indivíduos saudáveis sedentários pareados por sexo e idade (mediana (valor mínimo e máximo): 303 (76 a 1251) $\mathrm{kcal} / \mathrm{dia}$ vs 565 (214 a 1319) kcal/dia, respectivamente). Chama atenção que o percentual de contribuição do gasto energético para atividade física nos pacientes em HD é baixo, variando entre $10 \%$ e $16 \%$ do $\mathrm{GET}^{25,27}$, valor inferior ao esperado para a população em geral $(15 \%$ a $30 \%$ do $\mathrm{GET})^{12}$.

Em conjunto, esses resultados demonstram que pacientes com DRC apresentam atividade física reduzida. De fato, ao avaliar o nível de atividade física, definido pela razão entre GET/GER, mostrou-se que essa razão nos pacientes em HD (entre 1,3 e 1,4) era compatível com um estilo de vida sedentário ${ }^{26,27}$, segundo os pontos de corte propostos pela World Health Organization (WHO $)^{11}$. Corroborando esses achados, trabalhos anteriores já apontavam essa reduzida atividade física nos pacientes em HD em relação a indivíduos saudáveis, quando estudados por métodos indiretos, como pedômetro e acelerômetro 29,30 . Dessa forma, não há dúvida de que 
o sedentarismo é um achado comum em pacientes em hemodiálise. Estudos semelhantes em pacientes nos estágios não dialíticos da DRC, bem como em diálise peritoneal devem ser realizados para melhor conhecer o nível de atividade física nesses grupos específicos.

\section{Gasto energético total na DRC}

Poucos estudos buscaram avaliar o GET de pacientes com DRC, sendo que os realizados até o momento o fizeram por meio de sensores de movimento ${ }^{25,28}$. Baria et al. ${ }^{25}$ mostraram que o GET de pacientes em HD era menor que o de indivíduos saudáveis sedentários. Um estudo que avaliou a influência da inflamação no GET de pacientes em HD observou que, nos pacientes com condição clínica de inflamação, o GET ajustado por peso corporal foi significantemente menor quando comparado com o de pacientes sem inflamação $(25,5 \pm 4,1$ vs $32 \pm 6,7 \mathrm{kcal} / \mathrm{kg} / \mathrm{dia}$, $p<0,05$, respectivamente) e com indivíduos saudáveis $(25,5 \pm 4,1$ vs $32 \pm 6,6 \mathrm{kcal} / \mathrm{kg} / \mathrm{dia}, p<0,05$, respectivamente $)^{28}$. A ausência de trabalhos que tenham avaliado o GET dessa população por métodos mais precisos dificulta concluir se o mesmo se encontra alterado em pacientes com DRC com condição subclínica de inflamação.

\section{Equações de predição da TMB e do GET}

As equações de predição da TMB e do GET vêm sendo bastante empregadas na prática clínica para estimar as necessidades energéticas, uma vez que a aferição por calorimetria indireta é onerosa, além de requerer jejum e profissional treinado para a realização do exame.

No que se refere à estimativa da TMB, as principais equações utilizadas compreendem a de Harris \& Benedict ${ }^{31}$, a do documento da FAO/ OMS/UNU de $1985^{32}$ e a do mesmo documento publicado em $2001^{11}$ (Quadro 2). Há ainda outras equações também referenciadas em artigos científicos $^{33,34}$, mas menos empregadas ${ }^{35,36}$.

As equações de Harris \& Benedict $^{31}$, FAO$1985^{32}$ e FAO-2001 (ou de Schofield) ${ }^{11}$ foram desenvolvidas a partir de análise de regressões de estudos que avaliaram a TMB de diferentes populações. A equação de HB foi desenvolvida a partir de um banco de dados que incluía indivíduos adultos de ambos os sexos, atletas e não atletas, vegetarianos e não vegetarianos ${ }^{31}$. A equação do documento da FAO-1985 incluía indivíduos saudáveis, também de ambos os sexos, com diversas faixas etárias e de etnia predominante italiana ${ }^{32}$. $\mathrm{O}$ que diferenciou a equação proposta pela FAO-1985 32 da FAO-2001 ${ }^{11}$ foi a inclusão de uma

Quadro 2. Principais equações de predição da Taxa Metabólica Basal (TMB).

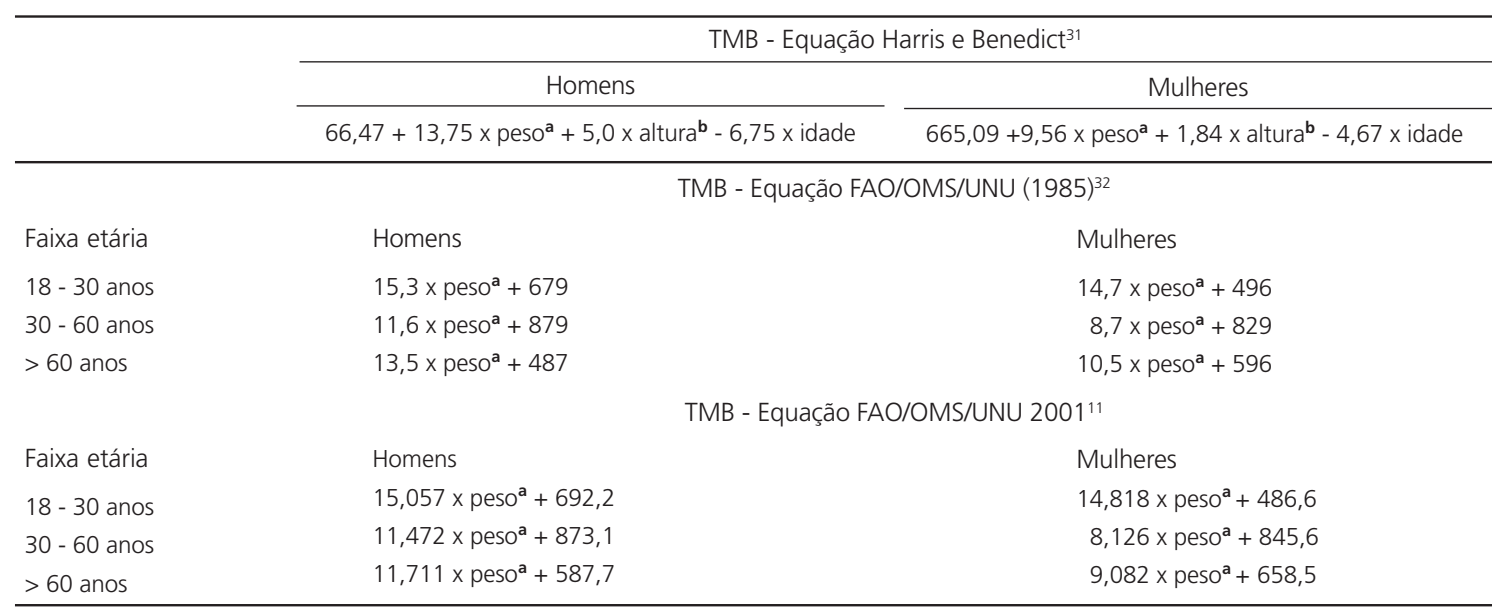

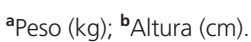

TMB: Taxa de Metabolismo Basal; FAO/WHO/UNU: Food and Agriculture Organization/World Health Organization/United Nations University. 
104 | JCD RODRIGUES et al.

amostra mais ampla com diversas etnias. Apesar de contemplarem amostras que incluíram indivíduos de ambos os sexos, com ampla faixa etária e grupo étnico, essas equações contêm variáveis específicas que as diferenciam ao estimar as necessidades energéticas. Como exemplo, a equação de HB inclui dados de idade, peso corporal e estatura do paciente ${ }^{31}$, ao passo que as equações da OMS não empregam esta última variável ${ }^{11,32}$, o que torna a utilização dessas duas equações mais simples na prática clínica. Vale enfatizar que as equações citadas foram desenvolvidas para estimar a TMB de indivíduos saudáveis. Logo, uma análise criteriosa deve ser realizada ao se empregá-las para pacientes com DRC. Até o momento, poucos são os estudos que avaliaram a precisão das equações que estimam a TMB nessa população.

Kamimura et al. ${ }^{37}$ buscaram avaliar se as equações de predição da TMB para indivíduos saudáveis são confiáveis para serem utilizadas em pacientes com DRC. Para tanto, o GER de 281 pacientes (124 em tratamento conservador, 99 em
HD e 58 em diálise peritoneal) e de 81 indivíduos saudáveis foi avaliado por calorimetria indireta e comparado com a TMB estimada pela equação de Harris e Benedict e FAO-2001. Notou-se que as equações superestimaram o GER aferido pela calorimetria indireta - tanto nos grupos de pacientes com DRC, como nos indivíduos saudáveis -, sendo a equação da FAO-2001 aquela que apresentou maior taxa de superestimação. Chama atenção que o erro obtido pelas equações era minimizado quando associado a algumas comorbidades (diabetes Mellitus, inflamação e hiperparatireoidismo), já que essas sabidamente aumentam o GER ${ }^{37}$. Contrapondo-se a esses achados, Lee et al. ${ }^{34}$ não observaram diferença significante entre o GER obtido pela calorimetria indireta e pelas equações de predição da TMB (HB, FAO-1985, FAO-2001 e outras) em 38 pacientes em diálise peritoneal. Apesar da ausência de diferença, notou-se que, nos pacientes super-hidratados, o GER mensurado pela calorimetria indireta encontrava-se subestimado em relação ao obtido pela equa-

Quadro 3. Estimativa do gasto energético total de acordo com o EBPG de nutrição.

\begin{tabular}{|c|}
\hline $\mathrm{GET}=\mathrm{TMB}(\mathrm{kcal} / \mathrm{dia}) \times \mathrm{NAF}^{\mathrm{a}}$ \\
\hline $\begin{array}{l}\text { a Classificação do estilo de vida em relação a intensidade da atividade física habitual, ou NAF recomendado pela OMS }{ }^{11} \\
\text { - Estilo de vida sedentário ou atividades leves: } 1,40 \text { a 1,69- Estilo de vida ativo ou moderadamente ativo: 1,70 a 1,99 } \\
\text { - Estilo de vida vigoroso ou vigorosamente ativo: } 2,00 \text { a 2,40 }\end{array}$ \\
\hline DRI de Energia ${ }^{12}$ \\
\hline Necessidade Estimada de Energia (NEE) \\
\hline $\mathrm{NEE}_{\text {Homens }}=662-\left(9,53 \times\right.$ idade $\left.^{\mathbf{a}}\right)+\mathrm{AF} \times\left(15,91 \times\right.$ Peso $^{\mathbf{b}}+539,6 \times$ altura $\left.^{\mathbf{c}}\right)$ \\
\hline NEE Mulheres $=354-\left(6,91 \times\right.$ idade $\left.^{\mathbf{a}}\right)+\operatorname{AF} \times\left(9,36 \times\right.$ Peso $^{\mathbf{b}}+726 \times$ alturac $\left.^{\mathbf{c}}\right)$ \\
\hline Onde a atividade física: \\
\hline $\begin{array}{l}\mathrm{AF}=1,00 \text { se NAF é estimado para ser } \geq 1,0<1,4 \text { (sedentário: trabalhos domésticos de esforço leve a moderado, caminhadas para } \\
\text { atividades do cotidiano e ficar sentado muitas horas por dia) }\end{array}$ \\
\hline $\begin{array}{l}\mathrm{AF}=1,11 \text { se NAF é estimado para ser } \geq 1,4<1,6 \text { (atividade leve: caminhadas }(6,4 \mathrm{~km} / \mathrm{h}) \text {, além das mesmas atividades do NAF sedentá- } \\
\text { rio) }\end{array}$ \\
\hline $\begin{array}{l}\text { AF }=1,25 \text { se NAF é estimado para ser } \geq 1,6<1,9 \text { (ativo: atividades aeróbicas, como corrida, natação e tênis, além das mesmas atividades } \\
\text { do NAF sedentário) }\end{array}$ \\
\hline $\begin{array}{l}\text { AF }=1,48 \text { se NAF é estimado para ser } \geq 1,9<2,5 \text { (muito ativo: ciclismo de intensidade moderada, corrida, pular corda, tênis, além das } \\
\text { mesmas atividades do NAF sedentário) }\end{array}$ \\
\hline
\end{tabular}


ção de HB, mas não em relação às equações da FAO-1985 e FAO-2001.

Com relação à estimativa do GET, o European Best Practice Guideline in Nutrition $(E B P G)^{8}$ propôs que se utilize uma das equações de predição da TMB, multiplicada pelo Nível de Atividade Física (NAF) proposto pela WHO. De maneira semelhante, a equação proposta pela DRI de energia ${ }^{12}$ para estimar as necessidades energéticas também inclui variáveis semelhantes à do EBPG na tentativa de individualizar o cálculo das necessidades energéticas (Quadro 3). Contudo, não há trabalhos que tenham avaliado a acurácia e precisão das propostas do EBPG e da DRI de energia para estimar o GET para pacientes com DRC.

Em resumo, esses trabalhos sugerem que o emprego de equações de predição para estimar a TMB de pacientes com DRC merece atenção, principalmente naqueles que clinicamente estáveis (ou seja, sem comorbidades que aumentem o GER), para os quais aquela medida parece estar superestimada. Portanto, para os renais crônicos sem comorbidades, a estimativa do GET, conforme proposto pelo EBPG, pode estar superestimada.

Outro importante ponto a ser questionado refere-se ao peso utilizado nessas equações, que poderia ser o atual ou o ideal/ajustado, sendo este último direcionado aos pacientes com baixo peso ou sobrepeso/obesidade. Igualmente, também se questiona qual o melhor método de escolha do cálculo do peso ideal/ajustado. Enfim, ainda são necessários mais trabalhos que respondam a essas perguntas para que se possa estabelecer a melhor equação de predição da TMB em pacientes com DRC.

\section{O N C L U S Ã O}

As recomendações energéticas propostas para pacientes com DRC variam entre $30 \mathrm{e}$ $40 \mathrm{kcal} / \mathrm{kg} / \mathrm{dia}$, conforme o guia de conduta empregado $2,7-9$. Contudo, tais recomendações não devem ser generalizadas a todos os pacientes com DRC, uma vez que os estudos que avaliaram o gasto energético dessa população mostraram que ele varia conforme a condição clínica, a modalidade de tratamento e o nível de atividade física.

O mesmo pode ser dito em relação às equações de predição da TMB, uma vez que sua acurácia e precisão dependem da condição clínica do paciente. Ademais, a ausência de trabalhos que tenham avaliado o gasto energético total de pacientes com DRC por métodos mais precisos dificulta estabelecer comparações para avaliar qual forma de estimar as necessidades energéticas apresenta melhor concordância com o gasto energético total medido. Sendo assim, até o momento não há trabalhos que possibilitem responder com precisão se as atuais recomendações de energia estão adequadas a todos os grupos de pacientes com DRC (exemplo: desnutridos, obesos, idosos, diferentes modalidades de tratamento e pacientes com comorbidades). Da mesma forma, a equação de predição da TMB a ser utilizada em pacientes com DRC ainda merece mais estudos até que a mais apropriada seja estabelecida.

Logo, considerando o exposto nesta revisão, independentemente do guia escolhido para cálculo das necessidades energéticas, é importante que essa estimativa seja utilizada como um ponto de partida para se fazer a prescrição da dieta. O acompanhamento periódico do estado nutricional é mandatório e deve ser feito de forma a possibilitar ajustes na ingestão energética para que o objetivo dietoterápico inicial seja alcançado.

\section{COLABORADORES}

JCDR e CMA trabalharam no levantamento das referências bibliográficas, redação e revisão do artigo. FL e CLO trabalharam na revisão do trabalho. CMA também orientou a redação do trabalho.

\section{REFERÊ NCIAS}

1. Cianciaruso B, Brunori G, Kopple JD, Traverso G, Panarello G, Enia G, et al. Cross-sectional comparison of malnutrition in continuous ambulatory peritoneal dialysis and hemodialysis patients. Am J Kidney Dis. 1995; 26(3):475-86. 
106 | JCD RODRIGUES et al.

2. National Kidney Foundation. K/DOQI - Clinical practice guidelines for nutrition in chronic renal failure. Am J Kidney Dis. 2000; 35(6 Suppl 2):S1-140.

3. Aparicio M, Cano N, Chauveau P, Azar R, Canaud $B$, Flory $A$, et al. Nutritional status of haemodialysis patients: a French national cooperative study. French Study Group for Nutrition in Dialysis. Nephrol Dial Transplant.1999; 14(7):1679-86.

4. Avesani CM, Carrero JJ, Axesson J, Qureshi AR, Lindholm P, Stenvinkel P. Inflammation and wasting in chonic kidney disease: partners in crime. Kidney Int. 2006; 70:S8-S13.

5. Kamimura MA, Draibe SA, Dalboni MA, Cendoroglo M, Avesani CM, Manfredi SR, et al. Serum and cellular interleukin- 6 in haemodialysis patients: relationship with energy expenditure. Nephrol Dial Transplant. 2007; 22(3):839-44.

6. Kramer HJ, Saranathan A, Luke A, Durazo-Arvizu RA, Guichan C, Hou S, et al. Increasing body mass index and obesity in the incident ESRD population. J Am Soc Nephrol. 2006; 17(5):1453-9.

7. Pollock C, Voss D, Hodson E, Crompton C. Caring for Australasians with Renal Impairment (CARI). The CARI guidelines. Nutrition and growth in kidneydisease. Nephrology (Carlton). 2005; 10(Suppl 5):177-230.

8. Fouque $D$, Vennegoor $M$, ter Wee $P$, Wanner $C$, Basci A, Canaud B, et al. EBPG guideline on nutrition. Nephrol Dial Transplant. 2007; 22(Suppl 2):ii45-87.

9. Cano NJ, Aparicio M, Brunori G, Carrero JJ, Cianciaruso B, Fiaccadori E, et al. ESPEN guidelines on parenteral nutrition: adult renal failure. Clin Nutr. 2009; 28(4):401-14.

10. Byham-Gray LD. Weighing the evidence: energy determinations across the spectrum of kidney disease. J Ren Nutr. 2006; 16(1):17-26.

11. World Health Organization. Human energy requirements. Rome: WHO; 2001. Technical Report Series; 2001.

12. Institute of Medicine. Food and Nutrition Board. Dietary reference intakes for energy, carbohydrate, fiber, fat, fatty acids, cholesterol, protein, and amino acids(Macronutrients). Washington (DC): National Academy Press; 2002.

13. Monteon FJ, Laidlaw SA, Shaib JK, Kopple JD. Energy expenditure in patients with chronic renal failure. Kidney Int. 1986; 30(5):741-7.

14. Schneeweiss B, Graninger W, Stockenhuber F, Druml W, Ferenci $\mathrm{P}$, Eichinger $\mathrm{S}$, et al. Energy metabolism in acute and chronic renal failure. Am J Clin Nutr. 1990; 52(4):596-601.

15. Ikizler TA, Wingard RL, Sun $M$, Harvell J, Parker RA, Hakim RM. Increased energy expenditure in hemodialysis patients. J Am Soc Nephrol. 1996; 7(12):2646-53.

16. Avesani CM, Cuppari L, Silva AC, Sigulem DM, Cendoroglo $M$, Sesso $R$, et al. Resting energy expenditure in pre-dialysis diabetic patients. Nephrol Dial Transplant. 2001; 16(3):556-65.

17. O'Sullivan AJ, Lawson JA, Chan M, Kelly JJ. Body composition and energy metabolism in chronic renal insufficiency. Am J Kidney Dis. 2002; 39(2): 369-75.

18. Avesani CM, Draibe SA, Kamimura MA, Dalboni MA, Colugnati FA, Cuppari L. Decreased resting energy expenditure in non-dialysed chronic kidney disease patients. Nephrol Dial Transplant. 2004; 19(12):3091-7.

19. Bazanelli AP, Kamimura MA, Silva CB, Avesani CM, Lopes MG, Manfredi SR, et al. Resting energy expenditure in peritoneal dialysis patients. Perit Dial Int. 2006; 26(6):697-704.

20. Kamimura MA, Draibe SA, Avesani CM, Canziani $M E$, Colugnati FA, Cuppari L. Resting energy expenditure and its determinants in hemodialysis patients. Eur J Clin Nutr. 2007; 61(3):362-7.

21. Cuppari L, Carvalho $A B$, Avesani CM, Kamimura MA, Dos Santos Lobao RR, Draibe SA. Increased resting energy expenditure in hemodialysis patients with severe hyperparathyroidism. J Am Soc Nephrol. 2004; 15(11):2933-9.

22. Utaka S, Avesani CM, Draibe SA, Kamimura MA, Andreoni S, Cuppari L. Inflammation is associated with increased energy expenditure in patients with chronic kidney disease. Am J Clin Nutr. 2005; 82(4): 801-5.

23. Wang AY, Sea MM, Tang N, Sanderson JE, Lui SF, Li $P K$, et al. Resting energy expenditure and subsequent mortality risk in peritoneal dialysis patients. J Am Soc Nephrol. 2004; 15(12):3134-43.

24. Ikizler TA, Pupim LB, Brouillette JR, Levenhagen DK, Farmer K, Hakim RM, et al. Hemodialysis stimulates muscle and whole body protein loss and alters substrate oxidation. Am J Physiol Endocrinol Metab. 2002; 282(1):E107-16.

25. Baria F, Kamimura MA, Avesani CM, Lindholm B, Stenvinkel P, Draibe SA, et al. Activity-related energy expenditure of patients undergoing hemodialysis. J Ren Nutr. 2011; 21(3):226-34.

26. Majchrzak KM, Pupim LB, Chen K, Martin CJ, Gaffney S, Greene JH, et al. Physical activity patterns in chronic hemodialysis patients: comparison of dialysis and nondialysis days. J Ren Nutr. 2005; 15(2):217-24.

27. Avesani CM, Trolonge S, Dele'aval P, Baria F, Mafra $D$, Faxe'n-Irving G, et al. Physical activity and energy expenditure in haemodialysis patients: an interna- 
tional survey. Nephrol Dial Transplant. 2012; 27(6): 2430-4.

28. Mafra D, Deleaval P, Teta D, Cleaud C, Arkouche $W$, Jolivot $A$, et al. Influence of inflammation on total energy expenditure in hemodialysis patients. J Ren Nutr. 2011; 21(5):387-93.

29. Johansen KL, Chertow GM, Ng AV, Mulligan K, Carey S, Schoenfeld PY, et al. Physical activity levels in patients on hemodialysis and healthy sedentary controls. Kidney Int. 2000; 57(6):2564-70.

30. Zamojska S, Szklarek M, Niewodniczy M, Nowicki M. Correlates of habitual physical activity in chronic haemodialysis patients. Nephrol Dial Transplant. 2006; 21(5):1323-7.

31. Harris JA, Benedict FG. A biometric study of basal metabolism in man. Washington (DC): Carnegie Institution of Washington; 1919.

32. World Health Organization. Energy and protein requirements. Geneva: WHO; 1985. Technical Report Series.

33. Frankenfield DC. On heat, respiration, and calorimetry. Nutrition. 2010; 26(10):939-50.
34. Lee SW, Kim HJ, Kwon HK, Son SM, Song JH, Kim MJ. Agreements between indirect calorimetry and prediction equations of resting energy expenditure in end-stage renal disease patients on continuous ambulatory peritoneal dialysis. Yonsei Med J. 2008; 49(2):255-64.

35. Fredrix EW, Soeters PB, Deerenberg IM, Kester AD, von Meyenfeldt MF, Saris WH. Resting and sleeping energy expenditure in the elderly. Eur J Clin Nutr. 1990; 44(10):741-7.

36. Mifflin MD, St Jeor ST, Hill LA, Scott BJ, Daugherty $S A$, Koh YO. A new predictive equation for resting energy expenditure in healthy individuals. Am J Clin Nutr. 1990; 51(2):241-7.

37. Kamimura MA, Avesani CM, Bazanelli AP, Baria F, Draibe SA, Cuppari L. Are prediction equations reliable for estimating resting energy expenditure in chronic kidney disease patients? Nephrol Dial Transplant. 2011; 26(2):544-50.

Recebido em: 24/2/2012

Versão final em: 20/7/2012

Aprovado em: 6/9/2012 
